\title{
NON-LINEAR SLIP-WEAKENING IN A ROTARY GOUGE FRICTION EXPERIMENT
}

\author{
G. Chambon ${ }^{1}$, J. Schmittbuhl ${ }^{1,3}$, and A. Corfdir ${ }^{2}$ \\ 1 Geology Lab., Ecole Normale Supérieure, Paris, France \\ 2 CERMES, ENPC/LCPC, Institu Navier, Champs sur Marne, France \\ 3 Institut de Physique du Globe, Strasbourg, France
}

\begin{abstract}
Two families of friction law are classically introduced to describe mechanical fault instabilities: slip weakening and rate and state friction laws. Generally opposed, we propose here, on the basis of our experimental results, to combine them in a single unified law. Using a large-displacement ring-shear apparatus for thick gouge samples (confinement: $0.1-0.5 \mathrm{MPa}$ ), we observe that slip-weakening largely dominates rate and state effects. The effective friction coefficient $\mu_{e f f}$ is found to undergo a power-law decrease with imposed slip $\delta$ : $\mu_{\text {eff }}=\mu_{0}+A \delta^{-\beta}$, with $\beta=0.4$. Although no characteristic length scale really exists, the main decrease of effective friction occurs over about $50 \mathrm{~cm}$ of slip. This appears quantitatively consistent with seismological data both in terms of typical weakening distances and characteristic rupture energies. Rate and state effects are involved over significantly smaller scales: $d_{c} \approx 100 \mu \mathrm{m}$. On the micro-scale, grain attrition exists. An image correlation technique reveals that the active interface is significantly wider than suggested by the microstructures. Slip-weakening appears caused by a progressive mechanical decoupling between a shear band and the bulk of the sample. Direct normal stress measurements along the shear interface have been possible and show a negligible influence of hoop stresses. Implications for faults are finally discussed.
\end{abstract}

\section{INTRODUCTION}

Real faults generally consist in complex tridimensional interfaces comprising thick layers of cataclastic gouge and damaged rocks (e.g. Chester and Chester [1]). In modeling studies, however, these thick structures are usually treated as perfectly thin interfaces, and their mechanical properties reduced to an effective friction law (e.g. Scholz [2]). The role of the friction law is to prescribe the evolution of the fault effective coeffi cient of friction as a function of the relevant physical parameters: slip, slip rate, asperity status, fault history, fault morphology, etc. In particular, the friction law should describe the physical mechanisms responsible for fault weakening during the initiation and development of seismic instabilities (earthquakes).

Two principal forms of friction laws coexist in the literature: the rate- and state-dependent friction (RSF) laws and the slip-weakening laws. In the RSF formulation, friction depends on the slip rate and on a set of variables characterizing the "state" of the frictional interface. This type of laws has been formulated on the base of numerous experimental results and appears to be applicable for a wide range of materials (Marone [3]). On the other hand, slip-weakening laws prescribe that the coeffi cient of friction essentially depends on slip displacement. They are less supported by experimental data than RSF laws (see nevertheless e.g. Ohnaka and Shen [4]), but are frequently employed for earthquake modeling owing to their relatively easy numerical implementation.

\section{EXPERIMENTAL SETUP}

Our experiments were performed in an annular, simple shear apparatus (Figure 1). The gouge sample is ring-shaped, with both height and width of $100 \mathrm{~mm}$ (inner radius $R_{1}=100 \mathrm{~mm}$, outer radius 
$R_{2}=200 \mathrm{~mm}$ ). Shear is applied by imposing a rigid rotation of the inner steel cylinder. As an analogy with faults, the displacement $\delta$ along sample inner boundary is called slip. Slip velocity $v=\dot{\delta}$ can be prescribed in the range $1.7-100 \mu \mathrm{m} . \mathrm{s}^{-1}$. A water cell connected to a pressure-volume controller is placed around this jacket and insures the radial confi nement of the sample $\sigma_{e}$.

Fault gouge is simulated in this study using an angular, quarry sand containing more than $99 \%$ of quartz. Working with submillimetric to millimetric particles, the thickness of our samples (perpendicular to the sliding direction) always exceeds 100 grains. This signifi cant thickness has to be contrasted with the narrower samples used in most other gouge friction experiments (e.g. Marone [3]).

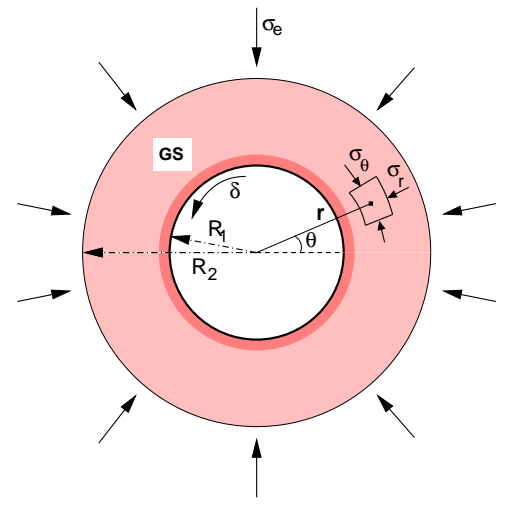

Figure 1: Sketch of the rotary setup (ACSA): The sample (GC) is sheared from the rotation of the inner cylinder of radius $R_{1}$ and confi ned $\left(\sigma_{e}\right)$ at a radius $R_{2}$ owing to a water cell.

The torque $\Gamma$ exerted by the driving system on the rotating cylinder is measured and converted in a shear stress $\tau$. Figure 2 shows a signifi cant weakening of shear stress $\tau$ with imposed slip. This weakening stops during (arbitrary long) hold periods at zero velocity, and proceeds as soon as shear is resumed. It is thus really slip-, and not time-, induced. We also see in Fig. 2a, that the amounts of slip required for a complete saturation of the shear stress drop are surprisingly large compared to grain size, typically about $0.5 \mathrm{~m}$. In Fig. 2a, we show that slip-weakening is not observed only during the initial shear (IS), but also, in a very reproducible way, during sense reversal (SR) and stress drop (SD) phases.

Figure $2 \mathrm{c}$ shows shear stress variations when prescribed velocity changes are imposed during a shear phase. The frictional strength of our synthetic gouge samples therefore includes velocity-weakening. Velocity-induced variations in shear stress, however, never exceed a few percents, and sometimes hardly emerge from the noise level. Velocity-weakening thus constitutes a second-order process compared to the major slip-weakening trend.

\section{NON-LINEAR SLIP WEAKENING}

Figure 3 shows that the post-peak decrease of shear stress $\tau$ with partial slip $\delta_{p}$ follows a linear path in log-log coordinates. This property appears particularly evident during initial shear phases: the linear decrease can then be observed over more than 2 slip decades. It holds generically, however, for all the shear phases that we studied, regardless of the preceding shear history and restrengthening 


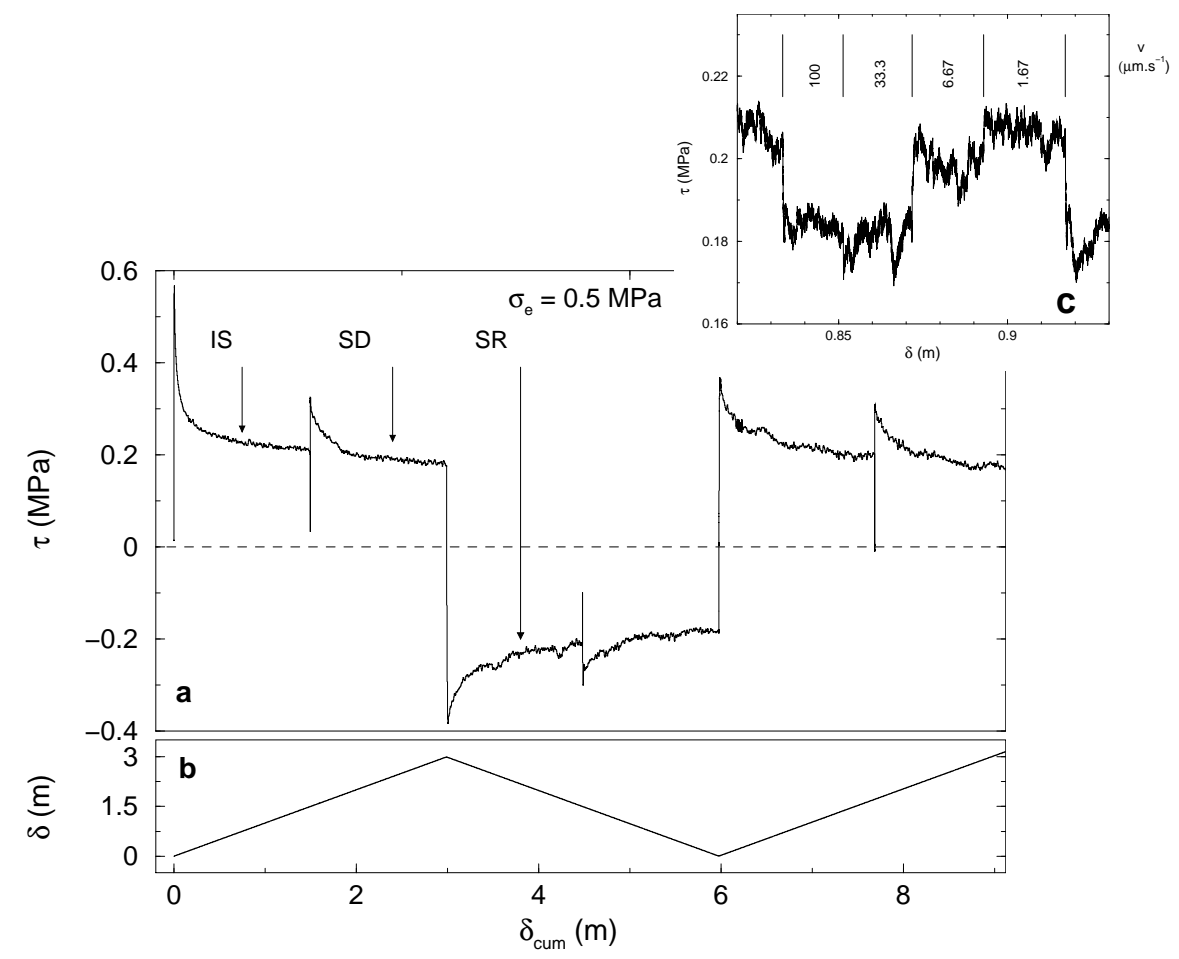

Figure 2: Response of the sample during successive shear phases: evolution of the shear stress (a), of the slip (b). Response to velocity changes is shown in (c).

events. The slip-weakening process can thus be modeled by a power law of the form:

$$
\tau\left(\delta_{p}\right)=\tau_{0}+\Delta \tau\left(\frac{\delta_{\star}}{\delta_{p}-\delta_{0}}\right)^{\beta},
$$

where, remarkably, the characteristic exponent $\beta$ is systematically equal to $0.4 \pm 0.05$.

\section{MICRO-STRUCTURAL OBSERVATIONS}

Two windows pierced in the apparatus enable to visualize the sample lower surface through a bottom glass plate. The observation zone is about $10 \times 10 \mathrm{~cm}$. A digital camera (resolution: $1536 \times 1024 \mathrm{px}$ ) has been installed below one of the windows to take series of pictures during the runs. Observations through the two windows pierced in the apparatus clearly reveal localization of deformation (Figure 4). Specifi cally, as soon as the peak strength is reached in a shear phase, grain displacements are found essentially confi ned to a narrow interfacial layer around the inner cylinder. In agreement with numerous other studies (e.g. Mülhaus and Vardoulakis [5]), the width of this shear band corresponds to 6-7-grain diameters. Furthermore, this width remains remarkably constant with ongoing shear, up to at least $37 \mathrm{~m}$ of cumulative slip.

In sand samples, strain localization appears associated with an active grain comminution process. As shown in the photo of Figure 4, imposed slip creates a fi ne cohesive powder which progressively 


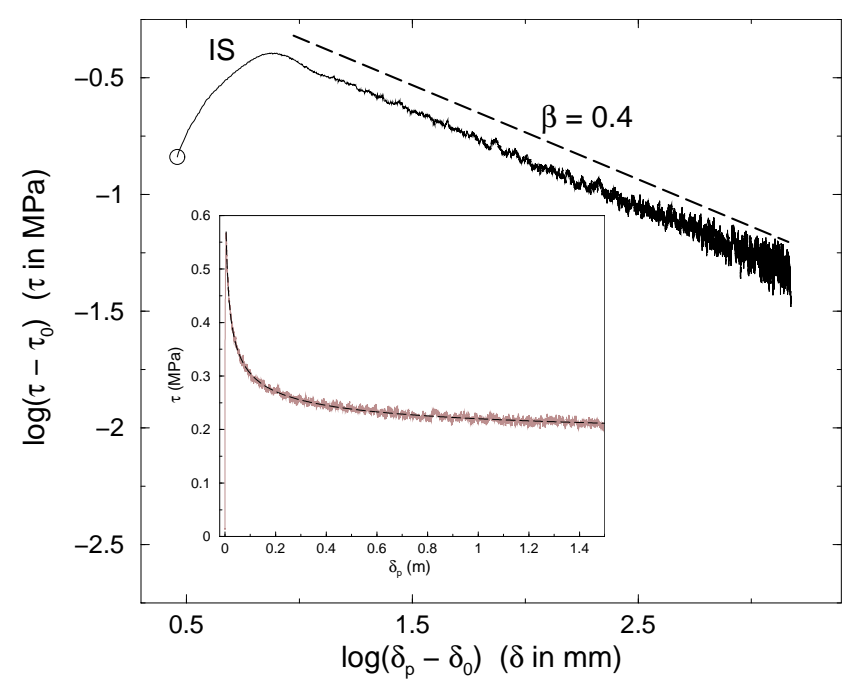

Figure 3: Power-law slip weakening during an initial phase in logarithmic scales. The inset shows the same data in a linear diagram together with the proposed fi t.

fi lls in the porosity of the shear band. We also observe that angularities of the initial particles tend to get rounded inside the band.

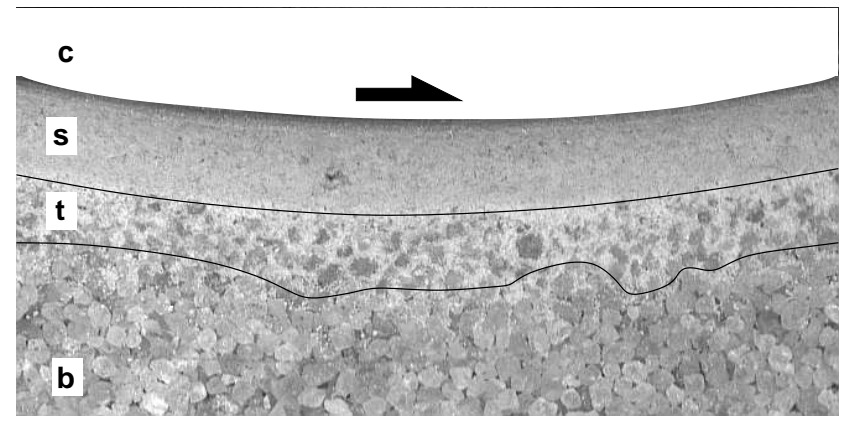

Figure 4: Micro-structures along the inner cylinder (c). A transition zone (t) separates the shear band (s) where particle flow is high and comminution intensive from the bulk (b). The scale is given by the particles which are $1 \mathrm{~mm}$ large.

In a recent paper (Chambon et al [6]), we presented results when Correlation Image Velocimetry (CIV) is applied to digital pictures. While measurements inside the shear band are corrupted by grain comminution, we were able to recover, with excellent accuracy, the local strain fi eld inside the bulk of the sample. Indeed, even once localization is established, the bulk remains submitted to slow and intermittent deformation. We identifi ed in particular a slow relaxation process active in the 10 or 20 particle-wide annulus surrounding the shear band. We showed that this slow relaxation actually corresponds to a slow and progressive decoupling between the shear band and the bulk of the sample. 


\section{FAULT IMPLICATIONS}

Regardless of their formulation, a recurrent concern with laboratory-derived friction laws is their quantitative discrepancy with natural, seismological data. Shear rupture energies $G_{c}$, in particular, hugely differ between friction laboratory tests $\left(10^{-2}-10^{0} \mathrm{~J} . \mathrm{m}^{-2}\right)$ and real earthquakes $\left(10^{6}\right.$ $10^{8}{\mathrm{~J} . \mathrm{m}^{-2}}^{-2}$ (Ohnaka [7]). Furthermore, this rupture energy is generally treated as a material parameter by classical friction laws, whereas various seismological results suggest that it does actually depend on earthquake size (Ide and Beroza [8]). In the same spirit, the characteristic slip scales involved in laboratory weakening mechanisms are about $10^{-6}-10^{-4} \mathrm{~m}$, while earthquake inversions usually yield weakening distances $D_{c}$ in the range $10^{-2}-10^{0} \mathrm{~m}$ (Guatteri and Spudich [9]).

In seismological studies, the slip-weakening processes active on faults during earthquakes are usually quantifi ed in terms of two linked parameters: the fracture energy $G_{c}$ and the characteristic weakening displacement $D_{c}$. To examine whether our laboratory results could be extrapolated to real faults, we computed the parameters $G_{c}$ and $D_{c}$ associated to the dominant slip-weakening process of our experiments.

The fracture energy $G_{c}$ is computed for each shear phase by integrating the $\tau$ versus $\delta_{p}$ relationship (Rice [10], Ohnaka [7]):

$$
G_{c}=\int_{\delta_{i}}^{\delta_{f}}\left[\tau(\delta)-\tau\left(\delta_{f}\right)\right] d \delta .
$$

where $\delta_{f}$ represents the partial slip at the end of the considered shear phase and $\delta_{i}$ is defi ned by: $\tau\left(\delta_{i}\right)=\tau\left(\delta_{f}\right)$ but before the peak stress. Typically we obtained $G_{c} \approx 2 \times 10^{4} \mathrm{~J}_{\mathrm{m}} \mathrm{m}^{-2}$ for $\sigma_{e}=$ $0.5 \mathrm{MPa}$. An essential feature of the power law slip-weakening exhibited in our experiments, is that $G_{c}$ does not constitute intrinsic material parameters. Integrating expression (1), one fi nds the following scaling relationship between the fracture energy $G_{c}$ and the quantity of slip $s$ undergone by the sample during a shear phase:

$$
G_{c} \sim s^{\alpha},
$$

where $\alpha=1-\beta=0.6$.

From a wide data compilation of seismological data, Ide and Beroza [8] showed that the energy radiated by earthquakes is roughly proportional to the seismic moment $M_{0}$ over more than 14 orders of magnitude in $M_{0}$. This observation, together with the classical result that earthquake stress drop is independent of $M_{0}$, indicates that the fracture energy should be proportional to earthquake slip: $G_{c} \sim s$. In an independent study, Abercrombie and Rice [11] directly evaluate the fracture energy of various earthquakes and end up with a similar, though slightly different, scaling relationship: $G_{c} \sim s^{1.3}$ (see Fig. 5). A scaling exponent of 0.6, as predicted from our experiments, would however be compatible with the data presented by Abercrombie and Rice [11] (see Fig. 5).

\section{References}

[1] F. M. Chester and J. S. Chester, "Ultracataclasite structure and friction processes of the Punchbowl fault, San Andreas system, California," Tectonophysics, vol. 295, pp. 199-221, 1998.

[2] C. H. Scholz, The Mechanics of Earthquakes and Faulting. 1997.

[3] C. Marone, "Laboratory-derived friction laws and their application to seismic faulting," Annu. Rev. Eath Planet. Sci., vol. 26, pp. 643-696, 1998.

[4] M. Ohnaka and L.-F. Shen, "Scaling of the shear rupture process from nucleation to dynamic propagation: Implications of geometric irregularity on the rupturing surfaces," J. Geophys. Res., vol. 104, pp. 817-844, 1999. 


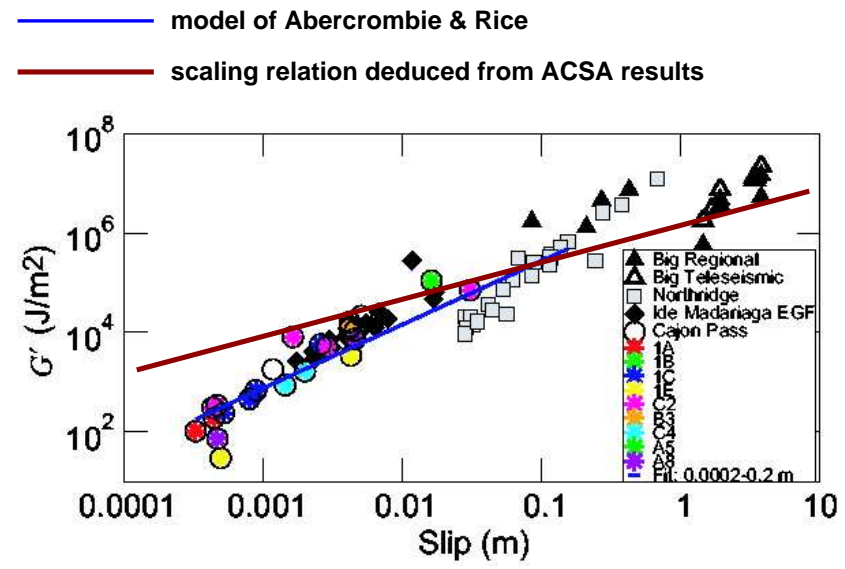

Figure 5: Scaling of the fracture energy after Aberbercrombie and Rice [11]. Superimposed is the fi t from our scaling: $G_{c} \sim s^{0.6}$.

[5] H. B. Mühlhaus and I. Vardoulakis, "The thickness of shear bands in granular materials," Géotechnique, vol. 35, no. 3, pp. 271-283, 1987.

[6] G. Chambon, J. Schmittbuhl, A. Corfdir, J.-P. Vilotte, and S. Roux, "Shear with comminution of a granular material: Microscopic deformations outside the shear band," Phys. Rev. E, vol. 68, 2003.

[7] M. Ohnaka, "A constitutive scaling law and a unifi ed comprehension for frictional slip failure, shear fracture of intact rock, and earthquake rupture," J. Geophys. Res., vol. 108, no. B2, 2003.

[8] S. Ide and G. C. Beroza, "Does apparent stress vary with earthquake size?" Geophys. Res. Lett., vol. 28, no. 17, pp. 3349-3352, 2001.

[9] M. Guatteri and P. Spudich, "What can strong-motion data tell us about slip-weakening faultfriction laws?," Bull. Seismol. Soc. Am., vol. 90, pp. 98-116, 2000.

[10] J. R. Rice, "The mechanics of earthquake rupture," in Physics of the Earth's Interior (A. M. Dziewonski and E. Boschi, eds.), pp. 555-649, Italian Physical Society/North Holland Publ. Co., 1980.

[11] R. E. Abercrombie and J. R. Rice, "Can observations of earthquake scaling constrain slip weakening?," Geophys. J. Int., 2003. submitted. 